\title{
Commentary: When balancing risks of thoracic endovascular aortic repair, renal dysfunction weighs heavily
}

Eric E. Roselli, MD, and Lars G. Svensson, MD, PhD

\footnotetext{
From the Department of Thoracic and Cardiovascular Surgery, Aorta Center, Heart and Vascular Institute, Cleveland Clinic, Cleveland, Ohio.

Disclosures: Authors have nothing to disclose with regard to commercial support.

Received for publication Feb 12, 2019; accepted for publication Feb 12, 2019; available ahead of print March 28, 2019.

Address for reprints: Eric E. Roselli, MD, Department of Thoracic and Cardiovascular Surgery, Cleveland Clinic, 9500 Euclid Ave, Desk J4-1, Cleveland, OH 44195 (E-mail: roselle@ccf.org).

J Thorac Cardiovasc Surg 2020;159:414-5

$0022-5223 / \$ 36.00$

Copyright (c) 2019 by The American Association for Thoracic Surgery

https://doi.org/10.1016/j.jtcvs.2019.02.056
}

Because we can, does not always mean that we should. Now that thoracic endovascular aortic repair (TEVAR) is readily accessible at most hospitals, there is an even greater need for improved understanding of the natural history of aortic disease. Providing personalized, tailored therapy requires a precise understanding of the balance between risk and reward. This includes a detailed assessment of the 3 main contributors to a successful outcome: condition-specific details (eg, aortic anatomy and pathology), patient-specific details (eg, nonaortic comorbidities such as renal dysfunction), and operator-specific details (eg, skill and experience, with documented outcomes; Figure 1).

The article from Brown and colleagues ${ }^{1}$ in this issue of the Journal highlights the importance of considering a patient's renal function when determining the value of providing TEVAR. They reviewed data from more than 27,000 Medicare patients during a 15-year period and assessed trends in patient selection, mortality, and morbidity in relation to renal dysfunction. Renal function was graded according to International Classification of Diseases, Ninth Revision codes for chronic kidney disease (I-IV, or endstage renal disease, with patients excluded who had the code "chronic kidney disease, unspecified").

Not surprisingly, renal dysfunction was associated with worse outcomes, and the effect correlated with the severity of dysfunction. This association was noted at 30 days, 1 year, and 5 years. In this series, 1-year survival for a patient on hemodialysis who underwent TEVAR was only $48 \%$, and at 5 years it was only $15 \%$. In an emergency situation, the benefit of TEVAR is clear, but in this series, fewer than $3 \%$ of patients underwent TEVAR for rupture. This competing risk of death is important to consider when deciding whether to perform a prophylactic aortic aneurysm repair. Cost analysis was not performed in this study, but TEVAR technology is expensive and should be used wisely. Furthermore, although TEVAR is less invasive, complications can be serious. The authors found that patients with worse renal function also experienced more in this data set.

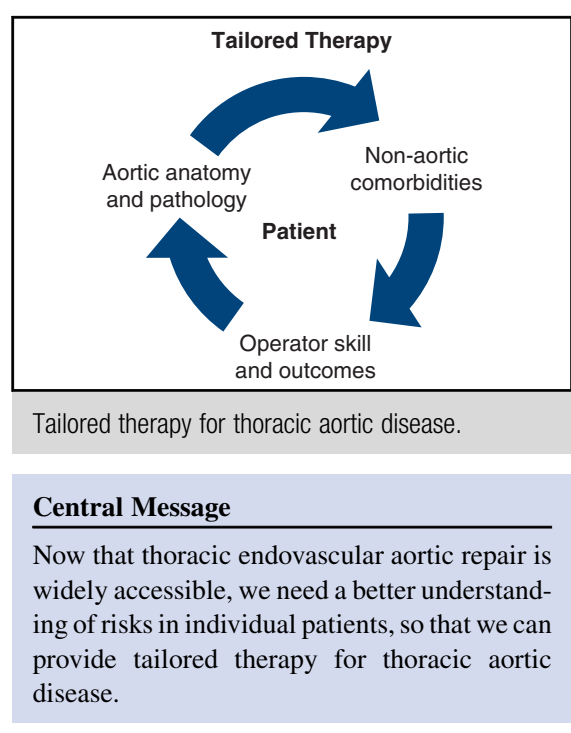

See Article page 402 .

complications like sepsis, respiratory failure, myocardial infarction, and paraplegia.

Brown and colleagues ${ }^{1}$ emphasize caution when considering TEVAR for a patient with severe chronic kidney disease or renal failure. When tailoring a treatment plan to an individual patient, however, one must be careful about applying data from this study to that patient's situation. As in any study using "big data," lack of granularity is a significant limitation of this administrative data set. It is important to recognize that these data are from the Medicare population, with an average age of 77 years. Details about the patients' anatomy or the morphology of their disease were not available, but it is interesting to note that more than $90 \%$ had aneurysm and not dissection as their main indication for repair. Data from this study may not apply to the younger patients we often see with acute or chronic aortic dissection and associated severe renal dysfunction, because they are not well represented

In this Medicare population of mostly patients with aneurysms, the predominant pathophysiology was atherosclerotic degeneration, a known risk factor for renal disease secondary to atheroembolization and renal artery occlusive disease, further complicating the generalization of these outcomes to a broader population of patients undergoing TEVAR. In most centers, the majority of patients requiring 


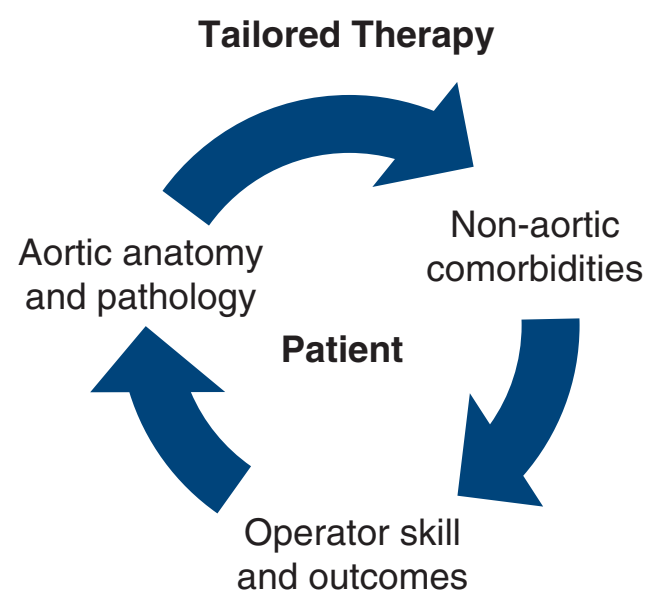

FIGURE 1. Tailored therapy for thoracic aortic disease.

TEVAR have dissection, and the average age of patients with dissection is younger than 65 years (ie, a nonMedicare population).
Finally, when developing a personalized, tailored treatment plan for patients with thoracic aortic disease, it is important to know the skill set, experience, and outcomes for one's own team. We learn from Brown and colleagues ${ }^{1}$ that, during the 15-year period of this study, confidence in treating elderly, high-risk patients with chronic kidney disease has improved. Devices and techniques have improved. Specialists who treat thoracic aortic disease have learned and taught others to provide more of this care to sicker patients. What we need is a better understanding of how to predict the risks of every treatment option in each individual patient so that we can provide personalized, precision aortic therapy and medical value. The study by Brown and colleagues ${ }^{1}$ provides some of this important information in one subset of patients, but we have a long way to go.

\section{Reference}

1. Brown CR, Chen Z, Khurshan F, Kreibich M, Bavaria J, Groeneveld P, et al Outcomes after thoracic endovascular aortic repair in patients with chronic kidney disease in the Medicare population. J Thorac Cardiovasc Surg. 2020;159:402-13. 